\title{
Dos crisis financieras y su impacto sobre el sistema bancario filipino: 1981-1987 y 1997-2000
}

DOI: $10.32870 /$ mycp.v3i11.111

Geneviève Marchini*

$\mathrm{E}$ $\mathrm{n}$ el transcurso de las dos últimas décadas, Filipinas experimentó dos severas crisis financieras que combinaron aspectos externos -dificultades en la balanza de pagos que empujaron a la devaluación de la moneda- e internos -operaciones fraudulentas y pérdida de confianza en el sistema financiero, crisis bancarias- y obligaron al país a emprender un proceso de ajuste. Al igual que México, Filipinas sufrió profundamente las consecuencias de la "crisis de la deuda externa" de los 80, y fue alcanzada en menor medida por la crisis asiática de 1997.

Las dos crisis comparten rasgos comunes: en primer lugar, estallaron en el contexto de una degradación del entorno macroeconómico internacional; la primera (1981-1987) se formó en el marco que propició la crisis de la deuda de las economías en desarrollo (bajo crecimiento mundial, tasas de interés internacionales altas y renuencia de la banca internacional a sostener su exposición al riesgo en estas economías), mientras la segunda lo hacía en el contexto del fenómeno de contagio que difundió la crisis asiática más allá de la economía inicialmente afectada (Tailandia). En segundo lugar, las circunstancias externas no explican enteramente estas crisis financieras, sino que adelantan su estallido y agravan sus consecuencias: en ambos casos, durante los años que antecedieron la crisis, aparecieron desequilibrios y se prendieron señales de alarma en el ámbito financiero interno, en parte atribuibles al paulatino proceso de liberalización aplicado al sistema desde los años 70 hasta la actualidad.

Ahora bien, las dos crisis difieren en un aspecto esencial: la primera causó una ruptura

* Investigadora del Departamento de Estudios del Pacífico de la Universidad de Guadalajara. bancaria de carácter sistémico, pues de 1981 a 1987 quebraron tres bancos comerciales, 32 instituciones de ahorro y 128 bancos rurales. Las autoridades tuvieron que rescatar el mayor banco comercial del país, el PND (Philippines National Bank), un importante banco de desarrollo, el DBP-Development Bank of the Philippines- (Nascimento 1991) y los activos de mala calidad adquiridos por la institución pública encargada del saneamiento del sistema, el APT (Asset Privatization Trust), superaron 20 por ciento de la cartera de crédito, alrededor de 18 por ciento del PIB (Klingebiel 2000). La segunda crisis no ha causado una situación de peligro financiero agudo y generalizado: si bien el PND volvió a caer en dificultades que requirieron su recapitalización, sólo quebraron hasta la fecha un banco comercial pequeño, siete instituciones de ahorro y 18 bancos rurales que representan menos de 1 por ciento de los activos del sistema (FMI 1999).

Este artículo se propone presentar estos dos episodios de crisis, enmarcándolos en el contexto del proceso de reforma financiera en curso durante todo el período considerado, y comparar sus consecuencias sobre el sistema bancario, la principal fuente de financiamiento de la economía. El estudio muestra un interés adicional: a diferencia de los demás países de la región, la esfera financiera de Filipinas tiene una problemática cercana a la mexicana y latinoamericana: estancamiento prolongado durante la "década perdida", bajo ahorro interno y crónica dependencia del financiamiento externo, importancia para la economía de las remesas de los trabajadores filipinos expatriados, sistema financiero relativamente pequeño y poco diversificado.

En una inicial sección se aborda el primer episodio de crisis, destacándose sus causas y su magnitud; en una segunda parte se presentan 
las medidas, tanto coyunturales como estructurales, que se tomaron para resolverlo. En una tercera sección se introducen los efectos de la crisis asiática sobre el sistema bancario, y en una última se concluye con algunas reflexiones comparativas.

\section{La crisis de 1981-1987}

La crisis que Filipinas empieza a experimentar a partir de 1981 se da al final de un ciclo de crecimiento elevado y de creciente vulnerabilidad financiera externa que este país compartió con las economías latinoamericanas víctimas de la "crisis de la deuda". Filipinas aplicaba políticas de desarrollo orientadas "hacia adentro", marcadas por una intensiva intervención del Estado, y desde la segunda mitad de la década de los 70 dependió fuertemente del ahorro externo para financiar una tasa de inversión más elevada, cercana a $30 \%$ del PIB. El deterioro del contexto económico internacional, que provocó en agosto de 1982 la moratoria mexicana, afectó a Filipinas, que experimentó una crisis de balanza de pagos en octubre de 1983. Al igual que en México y en América Latina, esta crisis significó el final del modelo de desarrollo por sustitución de importaciones y la introducción de reformas destinadas a fomentar la adopción de un esquema de crecimiento de tipo liberal. La transición económica se complicó en el caso filipino al coincidir a nivel político con el desplome del régimen dictatorial encabezado por F. Marcos.

Esta crisis en el sector externo no marcó, sin embargo, el inicio de los problemas financieros internos, sino una nueva etapa de profundización de una crisis inaugurada en enero de 1981, en el mercado de papel comercial. En esta fecha, el descubrimiento de una operación fraudulenta de gran magnitud provocó la pérdida de confianza y el retiro de los inversionistas, el colapso del mercado y la quiebra de las instituciones intermediarias. La fuga de los fondos hacia instituciones más seguras contaminó a su vez las porciones más débiles del sistema bancario: las instituciones de ahorro y los bancos rurales. En este contexto, la crisis de balanza de pagos de 1983, seguida de una declaración de moratoria sobre la deuda externa con la banca comercial, provocó pánico financiero y en especial una ola de "corridas" bancarias que afectó esta vez a la banca comercial, y se asoció a una fuga hacia las divisas y a salidas de capitales (Nascimento 1991).

Ahora bien, al intervenir después de un proceso de liberalización parcial de la esfera financiera, iniciado en la década anterior, la crisis de 1981-1987 puso en evidencia las fallas y debilidades de este proceso. Las autoridades filipinas eligieron una secuencia de liberalización financiera que se inició con una apertura parcial de la cuenta de capitales de la balanza de pagos, para proseguir con una desreglamentación, también incompleta, del sistema financiero interno. De esta manera, se relajaron desde los años 70 los controles de capitales facilitando la repatriación de la inversión extranjera directa, autorizando las compañías extranjeras a financiarse en pesos en el mercado local y legalizando la participación extranjera en el capital de los bancos filipinos. Asimismo, se fomentó la diversificación de cartera de los nacionales al crearse los depósitos en moneda extranjera y el sistema de unidades bancarias offshore. Los bancos autorizados a recibir los depósitos en divisas, cuyas tasas de interés eran libres, podían otorgar créditos en moneda extranjera a residentes, previo permiso del Banco Central, o convertidos en pesos, mediante acuerdo de swap con la autoridad monetaria. Los fondos así depositados podían trasladarse libremente fuera del país (ibid.).

Mientras se relajaban los controles sobre los flujos de fondos, tanto de nacionales como de extranjeros, hacia y desde las Filipinas, el sistema financiero interno siguió reglamentado durante toda la década de los 70. Los esfuerzos de las autoridades se centraron más bien en establecer condiciones de competencia más parejas entre los bancos, limitados por una reglamentación más severa, y los numerosos intermediarios no-bancarios, menos regulados (Abola 1999). Así, a la vez que el Banco Central ampliaba a partir de 1972 el rango de instituciones supervisadas para incluir los 
"cuasi-bancos" (instituciones no-bancarias que ofrecen sustitutos cercanos a productos bancarios como los depósitos), e introducía diversas medidas destinadas a volver menos atractivos estos instrumentos del mercado de dinero, relajaba algunas reglamentaciones prudenciales esenciales de la banca comercial, con el objetivo de incentivar su competitividad. Si bien se elevó el capital mínimo requerido para establecer un banco (a fin de favorecer la consolidación del sector y obtener instituciones más sólidas), las razones de capitalización (capital neto/ activos en riesgo) fueron rebajadas de 15 a 10 por ciento en 1973; las limitaciones puestas a la concesión de créditos a directores, accionistas e intereses relacionados fueron suavizadas y se abandonaron las reglamentaciones que regían las relaciones entre un banco y sus subsidiarias (Nascimento 1991).

La desreglamentación empezó solo al inicio de la década siguiente: en 1980 se buscó reducir la segmentación del mercado, introduciéndose la figura legal de la banca múltiple y redefiniéndose las operaciones autorizadas de la banca de ahorro. En el transcurso de 1981 y 1982, el Banco Central levantó todos los topes a las tasas de interés, y en 1982 empezó a disminuir paulatinamente los requisitos de reserva legal, de 20 por ciento a 18 por ciento, proceso que fue interrumpido por la crisis.

La apertura financiera temprana (que permitió entradas cuantiosas de fondos del exterior intermediadas por el sistema bancario), aunada a la relajación de las normas prudenciales en este sector, se combinaron para elevar rápidamente los fondos puestos a la disposición de la economía: en términos constantes (precios de 1980) la deuda de las empresas no-financieras (públicas y privadas) se cuadruplicó entre 1972 y 1981, y llegó a representar casi 60 por ciento del ingreso del sector. Una proporción elevada de este endeudamiento se hallaba denominada en dólares (más de 60 por ciento de la deuda empresarial en 1981-1982). El estallido de la crisis mostró después que este creci-miento muy dinámico del crédito y su dolarización habían suscitado una elevación insostenible del nivel de riesgo en el sistema. Después de 1983, la devaluación, el alza de las tasas de interés internas y el deterioro del contexto macroeconómico se conjugaron para elevar aún más la morosidad de los deudores bancarios (véase cuadro 1).

Ahora bien, esta evolución se debe tanto a las incongruencias en la secuencia de la liberalización financiera como a las condiciones de funcionamiento del sistema financiero en este período. Por un lado, los numerosos estudios publicados acerca de los procesos de liberalización financiera desde inicios de los 90 , y más aún, desde las crisis mexicana y asiática, muestran el carácter extremadamente riesgoso de una apertura temprana de la cuenta de capitales: en ausencia de un sistema financiero interno competitivo, eficiente y correctamente reglamentado y supervisado, y por lo tanto capaz de asignar los recursos productivamente, la mayoría de los analistas estiman que ésta se debe liberalizar de manera prudente y tardía. Ello implica que las reformas liberalizadoras internas y el reforzamiento de la supervisión y de la reglamentación (y no su relajamiento) deben anteceder la apertura de la cuenta de capitales.

En el caso de Filipinas, la estructura del sistema bancario favorecía la concentración de riesgos; en efecto, una proporción significativa del sistema bancario funcionaba bajo una 
estructura de holding financiera, en la cual diferentes instituciones financieras, bancarias y no-bancarias, podían coexistir con empresas no-financieras en el seno de un mismo grupo empresarial. Esta estructura constituía un terreno fértil para el otorgamiento de préstamos vinculados y fraudulentos, una práctica favorecida por la incertidumbre política que vivía el país y reforzada por la relajación de las exigencias prudenciales y por la incapacidad de los organismos de supervisión para hacer aplicar las reglamentaciones existentes. Por último, muchos créditos se otorgaban bajo criterios políticos y de "amiguismo"("crony capitalism"), lo cual explica también que el PNB, un banco comercial propiedad del Estado, y un banco de desarrollo público, el DBP, figuraban entre las instituciones más afectadas por la crisis.

\section{Rescate bancario y profundización de las reformas liberalizadoras}

Enfrentadas a una crisis que ponía en riesgo el sistema bancario en su conjunto, las autoridades tomaron las decisiones siguientes: no dejar quebrar instituciones de gran tamaño y propiciar más bien la absorción de empresas o intermediarios financieros privados insolventes por bancos públicos, proveer los recursos necesarios para las operaciones de saneamiento del sistema, puestas bajo el control del banco central, pues la agencia encargada de la protección de los depósitos, el PDIC (Philippines Deposit Insurance Company) no disponía de los recursos, ni humanos, ni materiales, requeridos para realizar semejante tarea (Nascimento 1991).

Para disponer de los activos bancarios de mala calidad, se creó una agencia pública específica, el APT, que estuvo en funcionamiento desde 1987. Esta agencia se estableció con el propósito de recibir, administrar y transferir rápidamente al sector privado los activos de mala calidad del sistema bancario. Adquirió a precio de libro, es decir, evitando al banco realizar pérdidas sobre estas ventas, un monto de activos estimado en casi $22 \%$ de los totales del sistema, en gran parte provenientes de los dos bancos públicos insolventes, el PNB y el
DBP, cuya cartera representaba, en $1985,36 \%$ del total del sistema, y se estimaba de mala calidad en la elevada proporción de $80 \%$ (Nascimento 1991). El costo total del rescate bancario fue muy elevado para las autoridades, si bien fue bastante menor en términos del PIB: en 1985 representó $20 \%$ del gasto agregado del gobierno (ibid.) y la situación del banco central se deterioró de tal manera que cayó en la insolvencia y requirió a su vez una operación de saneamiento financiero. Ahora bien, a pesar de los recursos invertidos, se considera que el ATP falló en cumplir sus objetivos. Hasta la fecha, más de la mitad de los activos que adquirió siguen sin venderse, esencialmente porque se trataba de créditos otorgados bajo criterios políticos o de manera fraudulenta (Klingebiel 2000). Más general, la operación de "limpieza" de los balances bancarios no logró evitar una severa contracción del crédito en los años que siguieron la crisis.

Paralelamente a las medidas de emergencia, las autoridades procuraron mejorar el funcionamiento del sistema financiero para evitar la reproducción de crisis similares. Estas medidas, tomadas bajo los gobiernos de Aquino y de Ramos, consistieron en liberalizar el sistema y en reforzar la reglamentación y supervisión del mismo. Las primeras medidas destacaron la reglamentación bancaria, estableciendo límites a la concentración de la cartera de créditos y eliminando el trato preferencial de ciertos grupos empresariales. Incluyeron también la transferencia de los programas de fomento y de crédito selectivo del banco central hacia programas financiados por el presupuesto o hacia bancos de desarrollo públicos (Abola 1999). Los requisitos de reserva legal se unificaron en 1989, y fueron rebajados más adelante para alcanzar 16\% en 1996 (Abola 1999). Sin embargo, es a partir de la década de los 90 que la liberalización del sistema se acelera, con una mayor desreglamentación de las transacciones corrientes y de los flujos de capitales con el exterior, y, en la esfera financiera interna, el fomento de la entrada de nuevos participantes nacionales desde 1995 , con una mayor presencia de la banca extranjera. Sin embargo, la liberalización no alcanzó plenamen- 
Cuadro 1

Cartera vencida del sistema bancario 1981-1999 (en porcentajes)

\begin{tabular}{lccccccccc}
\hline & 1981 & 1983 & 1985 & 1986 & 1993 & 1996 & 1997 & 1998 & $1999^{*}$ \\
\hline & & & & & & & & & \\
Cartera vencida/Cartera total & 13.2 & 8.9 & 16.7 & 19.3 & 5.3 & 3.5 & 5.4 & 11.0 & 14.5 \\
Banca comercial & 13.1 & 7.5 & 15.6 & 18.4 & 4.7 & 2.8 & 4.7 & 10.4 & nd \\
Banca de ahorro & nd & nd & nd & nd & 6.0 & 7.7 & 10.7 & 16.4 & nd \\
Banca rural & nd & nd & nd & nd & 21.3 & 14.1 & 15.4 & 18.0 & nd \\
\hline
\end{tabular}

* Información de octubre 1999.

Fuentes: Nascimento (1991), para los años 1981 a 1986; FMI (1999), anexo estadístico, para los años 1993-1998,

Bangko Sentral ng Pilipinas, News Release, 20/12/1999, http://www.bsp.gov.ph, para 1999.

te la asignación de recursos: hasta la actualidad, la banca debe destinar $25 \%$ a la agricultura y a la agroindustria, y $10 \%$ a las pequeñas y medianas empresas (FMI 1999). En 1993 el banco central es reestructurado y recapitalizado bajo el nuevo nombre de Bangko Sentral ng Pilipinas (BSP), adquiere independencia del poder político, concentrando sus actividades alrededor de la defensa del valor de la moneda nacional. Finalmente, la liberalización alcanzó también el sector de intermediarios nobancarios, que se abre a la inversión extranjera y los mercados financieros, con la fusión de las dos bolsas de valores en una nueva institución y la autorización de las casas de títulos extranjeras.

\section{La crisis de 1997: antecedentes y consecuencias}

Como producto de estas reformas, el número de instituciones de banca comercial se elevó de 35 en 1993 a 54 a fines de 1997 (BSP 1999). El sistema se halla dominado por un grupo de instituciones de gran tamaño: los diez bancos más grandes (entre los cuales figura una institución extranjera) representaban más de 55\% de los recursos del sistema (FMI 1999). Los niveles de capitalización mejoraron notablemente, alcanzando $19 \%$ para el sistema en su conjunto en 1993; su posterior disminución, vinculada del auge crediticio que precedió la crisis asiática, estableció esta razón en 16\% en 1996 (ibid., anexo estadístico). En efecto, el crédito al sector privado, que volvió a crecer a un ritmo moderado desde inicios de los 90, experimentó de nuevo una fuerte aceleración desde 1993-1994, con ritmos anuales promedio superiores a $30 \%$, triplicándose los pasivos del sector privado entre 1993 y 1997 (véase cuadro 2). Aun así, el grado de profundización financiera (o peso relativo de la esfera financiera en relación con el PIB), sigue siendo bastante más bajo en Filipinas que en el resto de Asia, y más cercano a niveles latinoamericanos.

$\mathrm{Al}$ igual que en el anterior episodio de rápido crecimiento bancario, una elevada proporción de la cartera estaba denominada en dólares, pues hasta la fecha, tanto las leyes fiscales como la ausencia de requisitos de reserva legal favorecen las operaciones bancarias en divisas: en 1997, los depósitos en moneda extranjera representaban más de la mitad de las obligaciones de los bancos, contra 3\% en 1990 (FMI 1999). Simultáneamente, la cartera vencida como proporción de la cartera total disminuía de $5.3 \%$ en 1993 a 3.5\% en 1996, sugiriendo que la expansión era globalmente sana (cuadro 1).

El estallido de la crisis asiática iba a confirmar el mayor grado de fortaleza del sistema bancario filipino, que resistió mejor que los de sus vecinos, ayudado, cabe subrayarlo, por menores y más tardíos flujos de capitales externos y por una menor concentración de los préstamos en una incipiente burbuja inmobiliaria (Alburo 1999). Sin embargo, la 
Cuadro 2

Préstamos del sistema bancario 1993-1998 (en billones de pesos filipinos)

\begin{tabular}{lrrrrrr}
\hline & 1993 & 1994 & 1995 & 1996 & 1997 & 1998 \\
\hline & & & & & & \\
Préstamos totales & & & & & & \\
Bancos comerciales & 567.0 & 722.0 & 980.4 & 1377.3 & 1753.6 & 1714.2 \\
Bancos de ahorro & 506.4 & 637.2 & 866.3 & 1221.8 & 1573.2 & 1542.5 \\
Bancos rurales & 45.1 & 65.7 & 89.2 & 122.1 & 139.6 & 130.5 \\
& 15.5 & 19.1 & 24.9 & 33.4 & 40.8 & 41.2 \\
\hline
\end{tabular}

Fuente: FMI (1999), anexo estadístico.

progresión de la cartera vencida ha sido continua: esta razón alcanzó más de $14 \%$ de los préstamos del sistema a fines de 1999. Esta alza de la morosidad, atribuible al impacto de la depreciación del peso y a una deficiente asignación de recursos, señaló, en opinión de las autoridades y de los organismos financieros multilaterales, la permanencia de debilidades asociadas a problemas de selección de los acreditados. Otros analistas, críticos de los programas de estabilización aplicados por el gobierno filipino, subrayan más bien la responsabilidad de estas mismas medidas, y en especial, del alza de las tasas de interés y de la consecuente recesión interna, en el deterioro de la situación financiera de los intermediarios. Esta vez, sin embargo, la insolvencia de instituciones fue menor, y fueron afectados en especial bancos pequeños (bancos de ahorro y bancos rurales), produciéndose también la recaída del $\mathrm{PNB}$, en cuyo capital sigue participando el estado filipino, y que se atribuye a la elevada exposición de su cartera a operaciones en divisas.

En concordancia con este diagnóstico, las autoridades filipinas tomaron medidas orientadas a "reforzar la capacidad de resistencia de los bancos a choques y a mejorar la habilidad de las autoridades a resolver la situación de bancos en dificultades" (FMI 1999). Éstas comprendieron esencialmente la elevación de las exigencias de capitalización y de creación de provisiones por pérdidas, la introducción de criterios más severos para la concesión de créditos (en especial en relación con empresas pertenecientes a un mismo conglomerado), la modernización de los métodos de supervisión, incluyendo la de los conglomerados, un reforzamiento de las prerrogativas y una mayor protección legal del BSP en los casos de bancos en problemas, con una mayor penalización de las instituciones que no cumplieran con las razones de capitalización exigidas.

Desde la crisis, el sistema bancario filipino ha iniciado una nueva fase de consolidación, que se ha producido entre los bancos comerciales, que dominan ampliamente el sistema (véase gráfica 1). Un solo banco comercial ha cerrado (Orient Bank) y se produjeron fusiones, motivadas en algunos casos por el reforzamiento de las normas de capitalización, que produjeron una elevación de la concentración del sistema. El gobierno emprendió la operación de saneamiento y de venta del PNB, enfrentando dificultades en el transcurso de este operación (desacuerdos con el mayor accionista del banco), que no le permitieron cumplir con el compromiso contraído con el FMI de enajenar esta institución en la primera mitad del año 2000. La banca extranjera, que representa actualmente $11 \%$ de los activos del sistema, está elevando su participación: tres adquisiciones han sido autorizadas recientemente mientras las autoridades examinan otra propuesta. Cabe señalar que estas negociaciones se han dado a pesar del deterioro del clima para la inversión extranjera en Filipinas en el transcurso de este año, debido al escándalo de corrupción al más alto nivel que ha sacudido desde inicios de año el mundo bursátil del país (The Economist, varias ediciones). 


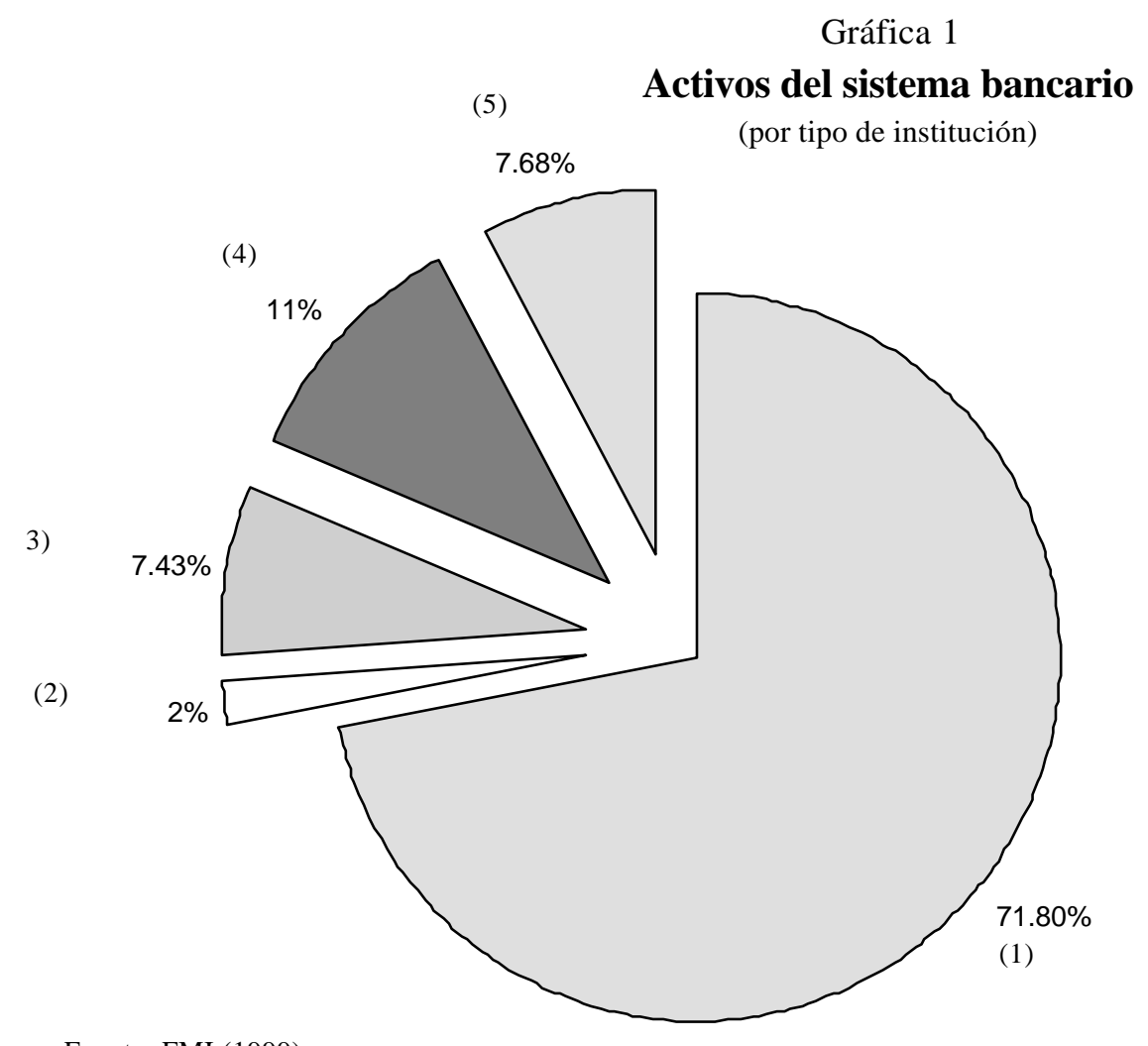

\begin{tabular}{|l|}
\hline (1) Banca múltiple \\
$\square(2)$ Banca rural \\
$\square(3)$ Banca de ahorro \\
$\square(4)$ Banca comercial \\
extranjera \\
$\square(5)$ Banca comercial
\end{tabular}

Fuente: FMI (1999)

\section{Reflexiones finales}

El estudio de las dos crisis financieras experimentadas por Filipinas en las décadas de los 80 y 90 sugiere algunas comparaciones, entre ambas crisis y entre éstas y la crisis mexicana de 1994-1995. En primer lugar, cabe señalar que en los tres casos, un movimiento de desreglamentación financiera interna y de simultánea apertura a los flujos transfronterizos de capitales terminó produciendo un excesivo endeudamiento de los agentes internos sustentado en gran parte en entradas a su vez insostenibles de fondos externos. En los tres casos, la asignación de recursos realizada por el sistema bancario interno resultó deficiente, aún cuando en la segunda crisis filipina el reforzamiento previo de la reglamentación permitió evitar la ola de insolvencia y el costoso rescate bancario que se dieron en 1981-1987 y en México.

El sistema financiero filipino presenta además otras características que vuelven útil su comparación con México, más allá del desarrollo relativamente atrasado de ambos sistemas: una de éstas está relacionada con la presencia de conglomerados que dificultan la supervisión de las actividades de las instituciones financieras, en razón de los vínculos estrechos que éstas mantienen con empresas nofinancieras, lo cual propicia la realización de operaciones riesgosas o fraudulentas, y la concentración de la cartera: estos problemas han estado presentes en la crisis mexicana de 198283 y se volvieron a plantear en 1994. Asimismo, los procesos de resolución de las crisis bancarias de 1981-1987 en Filipinas y de 1994-1995 en México han sido marcados por su costo elevado y su relativo fracaso (Klingebiel 2000).

Por otro lado, en ciertos aspectos, las soluciones aplicadas en Filipinas divergen: por ejemplo, México experimentó con los depósitos en divisas para residentes en bancos locales en los $70 \mathrm{y}$ ha luego restringido este sistema, mientras Filipinas lo ha vuelto a retomar, desarrollándose una extensa dolarización de los 
Gráfica 2

Tasas de interés 1993-1998

(\%, fin de período)

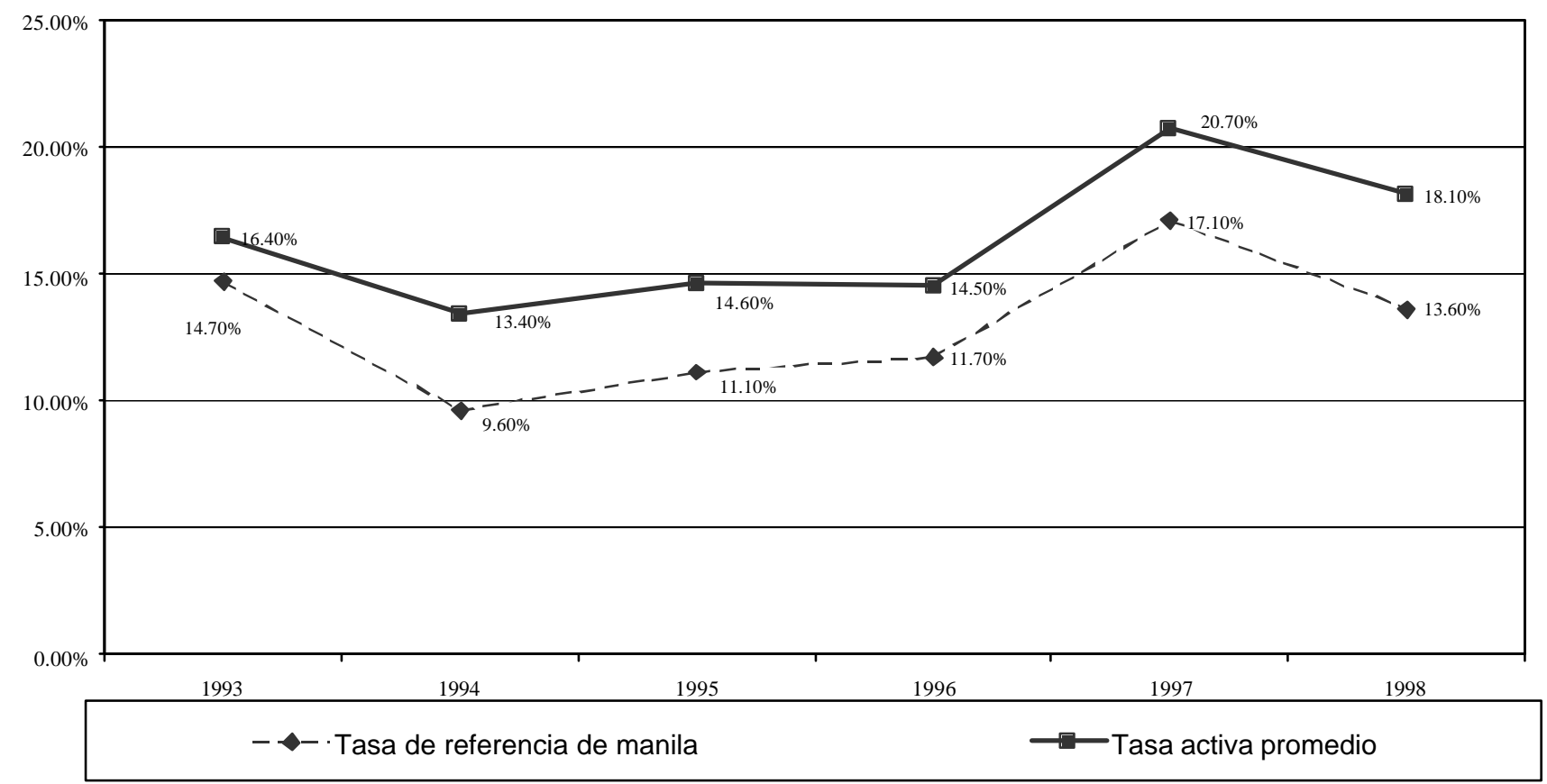

Fuente: FMI (1999), anexo estadístico.

depósitos bancarios. Asimismo, la banca filipina ha sido sujeta hasta la fecha a exigencias de crédito selectivo y a requisitos de reserva legal, mientras que México eliminó estos sistemas respectivamente en 1988-1989 y desde 19911992. En síntesis, el estudio comparativo de las diferentes soluciones aplicadas y la estimación de sus efectos ayudan a profundizar en la comprensión de los mecanismos que permiten a un sistema financiero apoyar o frenar el desarrollo económico de una nación.

\section{Fuentes}

Abola, Victor A. (1999) "Evolution of the Philippine financial system", en Seiichi Masuyama. Donna Vandenbrink \& Chia Siow Yue (eds.) East Asia's financial systems: evolution and crisis, Nomura Research Institute \& Institute of Southeast Asian Studies, Singapur; Alburo, Florián A. (1999) "The Asian financial crisis and the Philippines responses: long-run considerations", The Developing Economies, vol. XXXVII, núm 4, diciembre, p. 439-459; Bangko Sentral ng Pilipinas (1999)News Releases 1999, Información en línea, http://www.bsp.gov.ph; FMI (1999) "Philippines: selected issues", IMF Staff country report $n^{\circ} 99$ /92, agosto; Klingebiel, Daniela (2000) "The use of asset management companies in the resolution of banking crises", mimeo, Banco Mundial; Financial Times, información en línea, http://www.ft.com; Nascimento, JeanClaude (1991) "Crisis in the financial sector and the authorities reaction: the Philippines", en V. Sundararajan \& Tomás Baliño (eds.) Banking crises: cases and issues, FMI, 1991, pp. 175-233; The Economist, varias ediciones, año 2000. $T !:$ 